\title{
Visita técnica a uma clínica nefrológica realizada por estudantes de medicina: um relato de experiência
}

Technical visit to a nephrology clinic carried out by medical students: an experience report

Visita técnica a una clínica de nefrología realizada por estudiantes de medicina: un relato de experiencia

Gabriela Augusta Silva de Souza ${ }^{1}$, Grazielle Lima Silva ${ }^{1}$, Giovanna Mendes de Oliveira ${ }^{1}$, Mariella Prini Dias Lima ${ }^{1}$, Sâmia Marangoni Alarcon+1, Giullia da Costa1, Luana de Nathaly de Paulo, Vitória Fávero ${ }^{1}$, Mariana Sanches ${ }^{1}$, Angélica Nunes Rodrigues ${ }^{2 *}$.

\section{RESUMO}

Objetivo: Descrever o conhecimento e vivência de um estágio em uma clínica de nefrologia e o contato íntimo com a doença renal crônica. Relato de experiência: Baseado na visita em uma Clínica Nefrológica, na qual foi desenvolvida uma atividade com 16 discentes do curso de medicina, por meio de um estágio prático supervisionado por um médico nefrologista atuante na clínica em questão, a fim de compreender o funcionamento prático de um centro de referência em diálises, bem como ampliar o conhecimento da especialidade de nefrologia. Considerações finais: A participação no estágio extracurricular evidenciou que o envelhecimento populacional e o aumento de indivíduos com fatores de riscos durante os anos, tornaram-se fundamentais para formação, bem como para desenvolvimento de inúmeras habilidades médicas voltadas às doenças crônicas e suas consequências, além da notoriedade das profundas alterações na qualidade de vida dos indivíduos. Confirmando assim, a extrema necessidade da compreensão do funcionamento prático de um centro de referência em diálises, a fim de adotar melhores condutas num futuro próximo, além de propiciar um melhor entendimento sobre o biopsicossocial de pacientes portadores de doenças crônicas.

Palavras-chave: Doença renal crônica, Promoção de saúde, Prevenção de doenças.

\begin{abstract}
Objective: Describe the knowledge and experience of an internship in a nephrology clinic and intimate contact with chronic kidney disease. Experience report: Based on a visit to a Nephrology Clinic, which an activity was developed with 16 medical students, through a practical internship supervised by a nephrologist working at the clinic in question, in order to understand the practical functioning of a reference center in dialysis, as well as to expand the knowledge of the nephrology specialty. Final considerations: Participation in the extracurricular internship showed that population aging and the increase in individuals with risk factors over the years have become fundamental for training, as well as for the development of numerous medical skills aimed at chronic diseases and their consequences, in addition to the notoriety of the profound changes in the quality of life of individuals. Confirming the extreme need to understand the practical functioning of a dialysis reference center, in order to adopt better behaviors in the near future, in addition to providing a better understanding of the biopsychosocial aspects of patients with chronic diseases.
\end{abstract}

Keywords: Chronic kidney disease, Health promotion, Disease prevention.

1 Universidade de Franca (UNIFRAN), Franca - SP. *E-mail: nunesro@yahoo.com.br

2 Santa Casa de Misericórdia de Franca, Franca - SP. 


\section{RESUMEN}

Objetivo: Describir los conocimientos y experiencias de una pasantía en una clínica de nefrología y el contacto íntimo con la enfermedad renal crónica. Informe de experiencia: Basado en una visita a una Clínica de Nefrología, en la que se desarrolló una actividad con 16 estudiantes de Medicina, a través de una pasantía práctica supervisada por un nefrólogo que labora en la clínica en cuestión, con el fin de comprender el funcionamiento práctico de un centro de referencia en diálisis, así como ampliar los conocimientos de la especialidad de nefrología. Consideraciones finales: La participación en la pasantía extraescolar mostró que el envejecimiento poblacional y el aumento de individuos con factores de riesgo a lo largo de los años se han convertido en fundamentales para la formación, así como para el desarrollo de numerosas habilidades médicas dirigidas a las enfermedades crónicas y sus consecuencias, además de la notoriedad de los profundos cambios en la calidad de vida de las personas. Confirmando así la extrema necesidad de comprender el funcionamiento práctico de un centro de referencia de diálisis, con el fin de adoptar mejores comportamientos en un futuro próximo, además de proporcionar una mejor comprensión de los aspectos biopsicosociales de los pacientes con enfermedades crónicas.

Palabras clave: Enfermedad renal crónica, Promoción de la salud, Prevención de enfermedades.

\section{INTRODUÇÃO}

A Doença Renal Crônica (DRC) é conhecida a nível mundial como uma mazela de saúde pública. Pode ser definida como uma lesão que culmina na perda progressiva e permanente da função renal, além disso está associada diretamente ao envelhecimento como também na transição demográfica da população em geral. Frente a isso, as principais etiologias responsáveis pela DRC podem ser destacadas pela diabetes e hipertensão arterial. Assim, a literatura destaca que as doenças renais e bem como do trato urinário são responsáveis por volta de 850 milhões de mortes anual a nível global (SOUSA CND, et al., 2021). A DRC em fase mais avançada, pode também ser denominada fase terminal da insuficiência renal crônica, 0 aparelho renal não é capaz de realizar suas funções para a promoção da homeostase e por esse motivo, os pacientes são submetidos a um tratamento farmacológico e em algum momento do manejo, submetidos a terapia dialítica (WANDERSON AR, et al., 2021).

Desse modo, a DRC implica emrestrição alimentar demandando, assim, um cuidado maior, polifarmácia e dependência de acompanhamento do serviço especializado, seja a nível ambulatorial em seus estágios mais iniciais, seja na terapia renal substitutiva. Com a progressão da doença, os indivíduos acometidos podem apresentar declínio em seu estado de saúde, incluindo suas funções físicas como também psicossociais (ALMEIDA O, et al., 2019).

Atrelado a esse contexto, a Injúria Renal Crônica (IRC) pode ser representada como uma consequência da DRC, traz como apresentação uma redução total das múltiplas funções renais. Assim, uns dos principais e mais utilizados métodos de tratamento da IRC é a hemodiálise. Considerado um procedimento terapêutico naquelas que necessitam de Terapia Renal Substitutiva (TRS), que atua removendo catabólitos do organismo. O procedimento de TRS consiste em realizar hemodiálise ou a diálise peritoneal. A hemodíalise é executada ao conectar o paciente a uma máquina cicladora por meio de uma fístula arteriovenosa que realiza a limpeza e filtragem do sangue, bem como retirar sais e excesso de líquido, auxiliando no equilíbrio hidroeletrolítico. O procedimento mencionado é executado por volta três vezes na semana, em média quatro horas por sessão. No contexto da diálise peritoneal, pode-se destacar que o peritônio é utilizado como membrana de troca entre o corpo e o líquido dialisador, isso é possível devido a vasta rede de capilares e linfáticos na região, tal modalidade dialítica pode ser realizada a nível domiciliar e ambulatorial (SÔNIA CAP, et al., 2020).

Apesar de ser um procedimento que traz benefícios funcionais para o paciente, o tratamento também traz alguns danos, afetando diretamente a qualidade de vida, já que o paciente estará diante de uma doença incurável, dependente de uma máquina para sua sobrevida, além de alterações na própria imagem corporal bem como restrições alimentares e hídricas (SILVA MJ, et al., 2019). 
De acordo com a literatura vigente, a doença renal crônica vem apresentando uma alta distribuição mundial, com a prevalência estimada de $15 \%$ da população, principalmente em países portadores de recursos financeiros escassos. O diagnóstico precoce da doença é fundamental para que possa ser elaborado estratégias terapêuticas efetivas para prevenir ou até mesmo retardar a sua progressão, como também o ingresso do paciente na terapia renal substitutiva, sendo ela a diálise ou transplante renal. É possível constatar que até o ano de 2017 que $3 \mathrm{em}$ cada 100 brasileiros seriam portadores da doença em questão, como também, 5 em cada 10 mil pacientes se submeteriam a alguma modalidade dialítica (MARINHO AW, et al., 2020).

Dito isso, é de grande importância o cuidado médico com o paciente em hemodiálise, sabendo identificar e tratar as intercorrências clínicas associada ao procedimento, não raro, é comum tais pacientes apresentarem câimbras musculares, infecções de cateteres, náuseas, vômitos e arritmias, além de estarem sob risco de terem um maior número de internações e interações medicamentosas devido à polifarmácia (COITINHO D, et al., 2015). Diante da análise desse contexto, deve-se atentar também ao biopsicossocial dos pacientes em questão, devido a toda alteração de rotina e estresse que a hemodiálise acarreta, como perda de emprego, diminuição da autonomia, isolamento social e o medo da morte (BIANCA PS, et al., 2016). Portanto, nessa perspectiva, é fundamental o médico, além de manter-se sempre atualizado através da educação continuada, saiba ver o paciente além de sua doença, pois auxiliará na adesão do tratamento deste juntamente com a equipe multidisciplinar (MARIA JSS, et al., 2019).

Os Objetivos do Desenvolvimento Sustentável (ODS) da agenda 2030 (ODS 2030) foram criados pela Organização Mundial da Saúde (OMS), nela são contempladas 169 metas que deverão ser cumpridas até 2030. Dentre essas metas, destaca-se no objetivo três da agenda, a promoção de saúde e bem-estar, que consiste em intervenções integradas que contemplem o cuidado à saúde em todas as esferas do indivíduo, ao passo que ela é essencial ao fomento das capacidades humanas. Nessa perspectiva, tal meta foi incluída nesse contexto pelo fato de as doenças crônicas contribuírem para pobreza, privação dos mais vulneráveis e ser responsável por $60 \%$ de todas as mortes do mundo. Assim, até 2030 o objetivo é reduzir em um terço a mortalidade provocada por doenças crônicas não transmissíveis (OMS, 2013)

Diante do exposto e com base em tal questionamento, a experiência dos discentes durante a formação são essenciais para o aprimoramento da profissão, mostrando assima importância de ações de extensões, as quais aproximam os alunos das atividades práticas permitindo o desenvolvimento de senso crítico e reflexivo. Dessa forma, esse estudo teve como objetivo relatar a experiência vivenciada por discentes do curso de medicina através da Liga Acadêmica de Nefrologia, em uma visita técnica à uma clínicanefrológica especializada no cuidado do paciente renal em hemodiálise.

\section{RELATO DA EXPERIÊNCIA}

Trata-se de um relato de experiência baseado na visita de uma Clínica Nefrológica em abril de 2021, em horário extracurricular e localizada em uma cidade no interior de São Paulo. A atividade foi desenvolvida por 16 discentes do curso de medicina, por meio de um estágio prático supervisionado por um médico nefrologista atuante na clínica em questão. A vivência prática faz parte do cronograma de atividades desenvolvidas pelos alunos, juntamente com o centro acadêmico da universidade, através da Liga Acadêmica de Nefrologia, fundada e gerida pelos mesmos.

O estágio teve duração de duas horas e foi dividido em duas partes. Sendo assim, a primeira foi destinada a uma visita técnica pelas instalações da clínica, na qual o médico especialista intermediou. Num primeiro momento, fizemos uma discussão sobre os diversos tipos de diálise, diferenciando a diálise peritoneal e também a hemodiálise, em seguida, com esse embasamento teórico podemos conhecer o local reservado para execução das diálises, bem como o funcionamento de todo o maquinário, as salas destinadas para higiene e separação do material utilizado em cada sessão, foi possível também o contato com alguns pacientes enquanto realizavam o procedimento, podendo esclarecer quais eram as suas doenças de base, há quanto tempo frequentavam a clínica, quantas vezes por semana compareciam e o quanto a diálise causavam impacto em suas vidas. 
Num segundo momento, o médico nefrologista responsável conduziu uma palestra com o tema: "O que o médico generalista deve saber sobre nefrologia e doença renal crônica?". Nessa oportunidade, pudemos ter contato com a anatomia e fisiologia renal, foi explanado a respeito de como o médico não nefrologista deve triar e conduzir o paciente até um serviço nefrológico especializado, foi abordado alguns fármacos que agem diretamente nos rins, como por exemplo, os principais diuréticos usados para tratar doenças crônicas como a Hipertensão Arterial Sistêmica, que também tem repercussão na insuficiência renal. Nesse contexto, foi possível abordar a alta prevalência da doença renal crônica e seu impacto negativo na qualidade de vida do paciente e da família, como também para as organizações governamentais. Após a apresentação da palestra, abriu-se espaço para indagações para os discentes presentes.

Ao término das atividades, pode-se constatar o impacto que as doenças crônicas causam na vivência dos pacientes e também para a gestão de saúde pública, já evidenciado na literatura vigente, demonstrando, assim, a importância da promoção da saúde, atrelada com o manejo correto e diminuição da prevalência de tais comorbidades em nosso tecido social.

\section{DISCUSSÃO}

A idealização da visita deu-se a partir da ambição dos membros da Liga Acadêmica de Nefrologia de aprimorar os conhecimentos dos estudantes do curso de medicina acerca da DRC, de modo a auxiliar a aprendizagem individual, coletiva e interdisciplinar. A priori, ficou evidente durante todo o percurso que com o progressivo envelhecimento populacional e o aumento dos números de indivíduos com fatores de riscos durante os anos, a mesma passou a ser um trabalho árduo e um grande desafio para a saúde pública (SILVA PA, et al., 2020). Dessa forma, a participação extracurricular em estágios agrega na formação complementar e contribui com o desenvolvimento profissional e pessoal por meio da busca por novas experiências e aprendizados (FERREIRA IG, et al., 2016).

Ademais, durante a palestra ministrada, foi evidenciado a notoriedade das profundas alterações na qualidade de vida dos indivíduos, como por exemplo, as restrições alimentares e a sofrida terapia renal substitutiva (XAVIER V e LIMA CB, 2018). Visto que as taxas de incidência e prevalência de pacientes em diálise aumentam sucessivamente, o médico generalista deve incessantemente se atentar aos possíveis quadro clínicos e encaminhar ao especialista (THOMÉ FS, et al., 2019).

Assim, houve uma abordagem sobre a repercussão das mudanças da vida do paciente com doença crônica, o qual é privado de inúmeras oportunidades pessoais, interferindo assim na autoestima e nas relações interpessoais. Desse modo, se atinge determinada faixa etária, como a senescência, na qual a maior possibilidade de incapacidade física e mental pode causar mudanças bruscas e severas na saúde mental desses indivíduos (MARUYAMA ME e FERREIRA HG, 2020).

Para Malta DC, et al. (2017) a epidemia de Doenças Crônicas Não Transmissíveis (DCNT) resulta em consequências devastadoras acerca de todas esferas que compõe os indivíduos, como também para as famílias e comunidades que fazem parte, além de sobrecarregar os sistemas de saúde. Atrelado a isso, há unanimidade entre os autores pelo fato de que as DCNT afetam mais as populações desprovidas de recursos econômicos, pelo fato de estarem mais vulneráveis, mais expostas aos fatores de riscos e por possuírem menor acesso à rede de saúde, bem como as práticas de promoção à saúde e prevenção de doenças. A OMS salienta que pessoas portadoras das DCNT têm sua situação de pobreza agravada através de gastos familiares por conta da doença e também pela procura de serviços (MALTA DC, et al., 2017).

Analisando este contexto, deve-se lembrar dos objetivos criados pela OMS quanto ao desenvolvimento sustentável da agenda de 2030, esta compromete-se a não deixar ninguém para trás, abrangendo todas as pessoas que necessitam de cuidados e que vivem em uma situação de pobreza (ORGANIZAÇÃO DAS NAÇÕES UNIDAS (ONU), 2017). Sendo assim, indivíduos necessitados poderão ter cobertura universal de saúde, proteção de risco financeiro, acesso a serviços de saúdeessenciais de qualidade (CATANANTE GV, et al., 2017). 
Nesta perspectiva, Catanante GV, et al. (2017) destaca a importância de reforçar a atenção ao primeiro contato, pois corresponde ao livre acesso e usufruto do serviço pelo paciente que está sendo assistido para cada novo problema ou novo episódio de um problema em saúde. Isso garantirá um maior apoio para cada indivíduo que necessite de cuidados vitalícios.

Para isso, é necessário a postura com embasamento teórico e prático dos médicos generalistas nas abordagens com pacientes renais crônicos, bem como os dialíticos, para que saibam identificar possíveis fatores desencadeadores de repercussões, sejam esses alimentares ou medicamentosos e resolver essas questões para que aqueles possam ter melhor qualidade de vida, alcançando, dentre as limitações, o conceito de saúde proposto pela OMS, que pode ser definido como o alcance tanto do bem estar físico, quanto da plenitude do bem estar psicossocial, podendo enfatizar que saúde não é apenas a ausência de enfermidades (SILVA MJ, et al., 2019).

Desse modo, percebe-se a importância de acadêmicos de medicina a participarem e desenvolverem atividades extracurriculares para aprimorar e efetivar seus conhecimentos, aliando, assim, o conteúdo teórico com o que foi vivenciado na prática. Diante da análise desse contexto, fica evidente o entendimento acerca de como é a realidade de pacientes que vivem com as doenças de cunho crônico, em especial a Doença Renal Crônica. Com isso, espera-se que os futuros profissionais tenham postura ética e compromisso com cada ser humano que esteja sob seus cuidados, ajudando-os a entender sua doença e prover um atendimento que abrange o indivíduo como um todo, gerando um trabalho integrativo. Como reflexo disso, diante da criação de um vínculo sólido entre médico e paciente, pautado em confiança e acolhimento, o indivíduo que está sendo cuidado passa a aderir mais ao tratamento e consequentemente tendo a sua qualidade de vida substancialmente melhorada (SANTOS HM, 2017)

A vida acadêmica se contempla em muitas abordagens teóricas, dessa forma, a experiência permitiu aos discentes de medicina e futuros profissionais generalistas a compreensão do funcionamento prático de um centro de referência em diálises, bem como ampliar os olhares a uma especialidade pouco explorada na grade curricular do curso, que é a nefrologia, além de ter um maior contato acerca dos pacientes renais crônicos, determinando, assim, melhores condutas num futuro próximo, além de propiciar um melhor entendimento sobre o biopsicossocial de pacientes portadores de doenças crônicas, principais responsáveis pelas mazelas sociais na pós-modernidade.

\section{REFERÊNCIAS}

1. ALMEIDA AO, et al. Envolvimento da pessoa com doença renal crônica em seus cuidados: revisão integrativa. Ciênc. Saúde colet., 2019; 24 (5): 1689-1698

2. BIANCA OS, et al. Doença Renal Crônica: relação dos pacientes com a hemodiálise. Arquivos Brasileiros de Ciências da Saúde, 2016; 42 (1): 8-14.

3. CATANANTE GV, et al. Participação social na atenção primária à saúde em direção à agenda 2030. Ciênc. Saúde colet. 2017; 22 (12): 3965-3974.

4. COITINHO D, et al. Intercorrências em hemodiálise e avaliação da saúde de pacientes renais crônicos. Av Enferm. 2015; 33(3): 362-371.

5. FERREIRA IG, et al. Atividades extracurriculares e formação médica: diversidade e flexibilidade curricular. Interdisciplinary Journal of Health Education. 2016;1(2): 114-124.

6. MALTA DC, et al. Doenças crônicas não transmissíveis e a utilização de serviços de saúde: análise da Pesquisa Nacional de Saúde no Brasil. Rev Saude Publica. 2017;51 Supl 1:4s: 1s-10s.

7. MARIA JSS, et al. O Impacto do Tratamento Hemodialitico no Portador de Insuficiência Renal Crônico. Enciclopédia Biosfera, 2019; v.16 n.30: 419-433.

8. MARINHO AW, et al. Prevalência de doença renal crônica autorreferida em adultos na Região Metropolitana de Manaus: estudo transversal de base populacional. Epidemiol. Serv. Saude, Brasília, 2020; 29(1)

9. MARUYAMA ME, FERREIRA HG. Saúde mental e doenças crônicas em idosos de um grupo Hiperdia. REFACS (online); 2020; 8(Supl. 1): 599-611.

10. ORGANIZAÇÃO DAS NAÇÕES UNIDAS (ONU). Agenda 2030 para o Desenvolvimento Sustentável. Disponível em: https://www.unssc.org/sites/unssc.org/files/portuguese_2030_agenda_for_sustainable_development__kcsd_primer.pdf . Acessado em: 25 de agosta de 2021 
11. ORGANIZAÇÃO MUNDIAL DA SAÚDE (OMS). Universal eye health: a global action plan 2014. Disponível em: https://www.who.int/blindness/AP2014_19_English.pdf. Acessado em: 25 de agosto de 2021

12. SANTOS HM. Relatório final de estágio supervisionado I e II, Relatório apresentado pela Clínica de Psicologia da Universidade Tiradentes (UNIT) , 2017; 60 p.

13. SILVA MJ, et al. O conceito de saúde na Saúde Coletiva: contribuições a partir da crítica social e histórica da produção científica. Physis: Revista de Saúde Coletiva, Rio de Janeiro,2019; v. 29(1): 19 p.

14. SILVA MJ, et al. O impacto do tratamento hemodialitico no portador de insuficiência renal crônica. Enciclopédia biosfera, Centro Científico Conhecer - Goiânia, 2019; v.16 n.30; p. 419.

15. SILVA PA, et al. Política pública brasileira na prevenção da doença renal crônica: desafios e perspectivas. Revista de Saúde Pública 2020, 54: 6 p.

16. SÔNIA CAP, et al. A comparação do desempenho ocupacional entre as modalidades de tratamento dialítico. Cadernos Brasileiros de Terapia Ocupacional 2020, v.28 n.4, 1220-1233

17. SOUSA CND, et al. Percepção de portadores de doença renal crônica sobre o tratamento hemodialitico, 2021, № 64: 5594 - 5598.

18. THOMÉ FS, et al. Inquérito Brasileiro de Diálise Crônica 2017. J. Bras. Nefrol. 2019; 41 (2): 208-214.

19. WANDERSON AR et al. Repercussões da hemodiálise no paciente com doença renal crônica: uma revisão da literatura. Revista Pró-UniverSUS, 2020, v.11 n.1: 88-97

20. XAVIER V, LIMA CB, Tratamento da doença renal crônica: abordando as contribuições da teoria do autocuidado, Temas em saúde 2018, Vol. 18 № 1: 305-323. 